\title{
Unraveling the complexities of gut endocrinology
}

\author{
Daniel J Drucker
}

Few endocrinologists think of the gut as a complex endocrine organ, unless they encounter patients with carcinoid tumors or a gastrinoma localized to the duodenum. Gut hormone deficiencies caused by partial or complete loss of enteroendocrine cell function are even less common, but recent findings hint at their importance.

In 2006, Wang J et al. (N Engl J Med 355: 270280) described three children with inactivating mutations in neurogenin 3, a transcription factor important for endocrine cell development in the pancreas and gut. All three children presented with vomiting, metabolic acidosis, and malabsorptive diarrhea during the first few weeks of life, which required parenteral nutrition. Small-bowel biopsies revealed an almost complete absence of gut enteroendocrine cells. This novel endocrine syndrome is characterized by complete loss of enteroendocrine cell products that are critically important for control of nutrient absorption and energy homeostasis.

A similar clinical presentation occurs in humans with an inactivating mutation in neuroendocrine convertase 1 (NEC1, formerly PC1) - which is required for bioactivity of peptides such as glucagon-like peptide 1 (GLP-1). Patients present with varying degrees of small-bowel absorptive dysfunction, and defects in the processing of other endocrine peptides. The precise identity of the peptide hormones required for optimal control of nutrient absorption that are affected by loss of NEC1 activity in these patients is not yet clear, but analysis of NEC1-inactivating mutations in mice and humans reveals a broad spectrum of NEC1 substrates.

The importance of the enteroendocrine system in control of energy and glucose homeostasis might be revealed through subtle perturbations of gut endocrine cell function, rather than complete ablation of all enteroendocrine cell populations. Individuals with specific polymorphisms in TCF7L2 (which encodes transcription factor 7 -like 2) exhibit increased
Subtle defects in enteroendocrine cell function might be risk factors for the development of $\beta$-cell failure and, ultimately, clinical diabetes

DJ Drucker is the Director of the Banting and Best Diabetes Centre, a Scientist in the Samuel Lunenfeld Research Institute and Staff Physician at the Mount Sinai Hospital, and a Professor of

Medicine at the University of Toronto, Toronto, Canada.

\section{Competing interests}

The author has declared associations with the

following companies: Amylin Pharmaceuticals, Arisaph

Pharmaceuticals, BristolMyers Squibb, Conjuchem, Eli Lilly, GlaxoSmithKline, Merck, Novartis, and Transition Pharmaceuticals. See the article online for full details of the relationship.

www.nature.com/clinicalpractice doi:10.1038/ncpendmet0461 prevalence of type 2 diabetes. This transcription factor is highly expressed in the gut, including in enteroendocrine L cells, where it controls transcription of the proglucagon gene (and hence GLP-1 production). Reduced activity of transcription factor 7-like 2 might be associated with defective GLP-1 production and/or impaired $\beta$-cell function, and an increased risk of diabetes, in individuals with specific TCF7L2 alleles. Data published by Florez JC et al. in 2006 (N Engl $\checkmark$ Med 355: 241-250) extend these findings; this paper analyzed the relationship between TCF7L2 genotype and risk of developing diabetes observed in the Diabetes Prevention Program study. Individuals with specific TCF7L2 variant alleles progressed more frequently from impaired glucose tolerance to diabetes during the 3-year study. Furthermore, those with the high-risk TT genotype exhibited reduced insulin secretion, consistent with reduced secretion of one or more gut-derived factors (e.g. GLP-1), perhaps associated with impaired $\beta$-cell function and increased risk of developing diabetes. These findings, and data that demonstrate reduced meal-stimulated GLP-1 secretion in individuals with type 2 diabetes, suggest that subtle defects in enteroendocrine cell function might be risk factors for the development of $\beta$-cell failure and, ultimately, clinical diabetes.

Collectively, these loss-of-function mutations highlight the importance of slight or substantial defects in enteroendocrine cell function for the control of nutrient absorption and glucose homeostasis, and provide new mechanistic insights. Therapeutic administration of gut hormones and pharmacological enhancement of gut hormone action are receiving increased attention as potential new therapies for the treatment of obesity or diabetes. The re-emerging importance of gut hormones in common endocrine disorders firmly establishes the gut endocrine system as an essential component of physiological systems that regulate nutrient intake, absorption, and metabolic homeostasis. 